\title{
Development of a Navigator and Imaging Techniques for the Cryogenic Dark Matter Search Detectors
}

\author{
Chris Wilen \\ Office of Science, Science Undergraduate Laboratory Internship (SULI) \\ Carleton College \\ KIPAC SLAC National Accelerator Center \\ Menlo Park, CA
}

August 13, 2010

Prepared in partial fulfillment of the requirements of the Office of Science, Department of Energy's Science Undergraduate Laboratory Internship under the direction of Richard Partridge at the Stanford Linear Accelerator Center.

Participant:

Signature

Research Advisor:

Signature 


\section{TABLE OF CONTENTS}

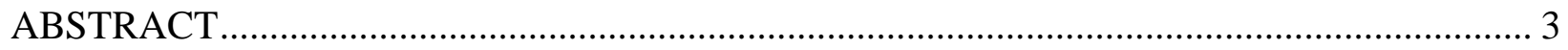

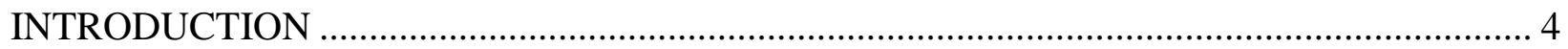

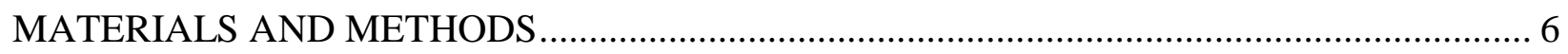

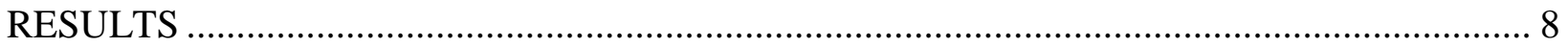

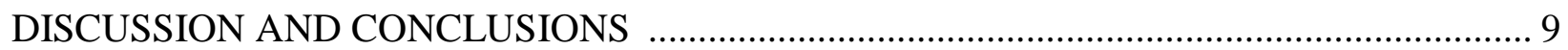

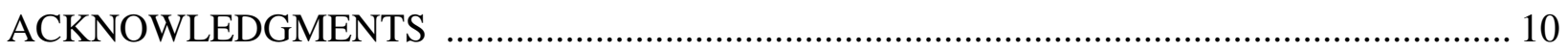

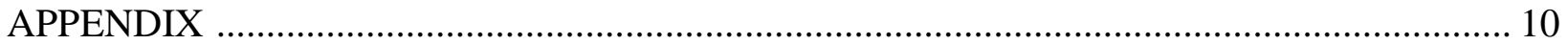

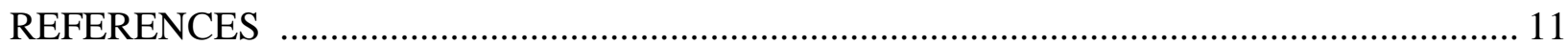

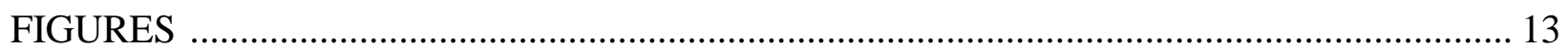

ACKNOWLEDGMENTS 


\begin{abstract}
Development of a Navigator and Imaging Techniques for the Cryogenic Dark Matter Search Detectors. CHRIS WILEN (Carleton College, Northfield, MN 55057) RICHARD PARTRIDGE (Stanford Linear Accelerator Center, Stanford, CA 94025)
\end{abstract}

This project contributes to the detection of flaws in the germanium detectors for the Cryogenic Dark Matter Search (CDMS) experiment. Specifically, after imaging the detector surface with a precise imaging and measuring device, we developed software to stitch the resulting images together, applying any necessary rotations, offsets, and averaging, to produce a smooth image of the whole detector that can be used to detect flaws on the surface of the detector. These images were also tiled appropriately for the Google Maps API to use as a navigation tool, allowing viewers to smoothly zoom and pan across the detector surface. Automated defect identification can now be implemented, increasing the scalability of the germanium detector fabrication. 


\section{INTRODUCTION}

The effects of dark matter have been observed throughout the last century on astrophysical scales. Fritz Zwicky was the first to discover evidence of dark matter when he observed the Coma cluster of galaxies in 1933. By looking at the overall brightness of the cluster, and counting the galaxies, he was able to estimate how much mass was contained in the cluster. Observing the outer galaxies, however, Zwicky found his estimate did not match the mass given by Keplerian motion. The difference between the two estimates turned out to be almost 400 times[1], indicating the existence of this unseen mass was a significant result.

Since then, various theories such as modified gravity have been tested, but the one that held up most successfully was that astrophysical structures such as the Coma Cluster were filled with something massive but invisible to our detection methods, named dark matter. Many experiments searching for dark matter have been performed by looking at the movement of galaxies. Evidence for dark matter lies in our own spiral galaxy, which, when measuring the rotation curve by looking at the maximum redshift at different angles, levels out instead of dropping off as would be expected from the visible matter (Figure 1). Gravitational lensing also provides strong evidence for the existence of this invisible, "dark" matter, when light is bent more than it would be from visible matter.

Other observations give indications about the nature of dark matter. It often clusters around and throughout galaxies and other visible matter, so it clearly interacts with visible matter through gravity. Evidence suggests that the interaction with dark matter does not go much further than gravity. One of the strongest arguments for dark matter comes from the recent analysis of the Bullet Cluster (Figure 2). X-ray imaging shows two galaxy clusters colliding in the center, but gravitational lensing shows two concentrations of matter to either side, leading to the conclusion that the clumps of dark matter in each of the clusters went through each other with little interference. This gives strong evidence against modified 
gravity theories, which cannot explain this effect[2]. Given dark matter's small cross section and the fact that it is unable to interact electromagnetically with photons, the limits of interaction can be narrowed to dark matter interacting with itself only through gravity and possibly the weak force.

Current estimates based on NASA and the Wilkinson Microwave Anisotropy Probe (WMAP) survey predict that the universe is made of roughly $4.6 \%$ atomic matter that we experience, $23 \%$ dark matter, and $72 \%$ dark energy $[3,4]$. Dark matter is significant, making up $85 \%$ of matter, and yet we know little about its characteristics. The Cryogenic Dark Matter Search experiment aims to detect it directly on a much smaller, single particle scale, instead of looking only at its gravitational effects in large masses. Specifically we will be looking for interactions between a type of (non-baryonic) dark matter called Weakly Interacting Massive Particles (WIMPS) and atomic matter in germanium detectors.

Because dark matter does not interact electromagnetically, it is assumed to be constantly flowing through the matter around us, unnoticed by any current detectors. For a significant interaction to occur, the WIMPs have to hit the nucleus of the atoms themselves, transferring energy to the atom. For the detector in this experiment, we picked germanium over silicon and other more widely used options because of its high cross section (more area to interact with the dark matter and a higher density leads to a greater probability of detection). When a WIMP interacts with the a germanium nucleus, some energy is transferred to the germanium from the dark matter particle through nuclear recoil. This energy propagates through the germanium in the form of phonons (lattice vibrations) until it reaches patterns of Transition Edge Sensors (TES), located on either side of the germanium crystal.

When they enter the aluminum in these sensors, the phonons break apart some of the cooper pairs (the superconducting carriers), and the resulting quasiparticles are absorbed into a tungsten strip (Figure 3). The whole detector is kept at several milliKelvin, right on the edge of superconductivity for tungsten. As the resistivity of the tungsten changes due to 
the quasiparticiles, a negative feedback loop reduces current through the tungsten to keep it at the same temperature. This change in current is measurable and recorded as a WIMP detection.

We needed to be able to distinguish dark matter detections from background radiation and decays of particles around the detector. When a charged particle or other ionizing radiation goes through the germanium, its electric field rips off electrons. By looking at the different signatures of charge build up on small electrodes patterned on surface of the detector, we were able to eliminate detections that looked like decays from atomic matter nearby or electron recoil, and minimize background.

The photolithography used for patterning these sensors on the germanium crystals is much more expensive and error-prone than similar processes for silicon. As a result, a method of reviewing the detectors in each stage of the patterning process, looking at them in detail, and finding errors needs to be established. I developed software to take images of the detector and piece them together into larger tiles that can be processed to find errors. I also created an online navigator to examine these images with.

\section{MATERIALS AND METHODS}

We used a precise imaging and measuring device made by Optical Gauging Products (OGP) to help with the imaging process. Using several points on the detector, we established a coordinate system corresponding with that of the detector for the camera to move along. First, the z-value of three points was taken using adjustment of focus. With a plane representing the surface of the detector created using these points, we then defined the $\mathrm{x}$ and $\mathrm{y}$ axis, zeroed around a point in the center and lined up with the detector's vertical charge-detecting electrodes. Throughout the CDMS experiment, several detector patterns have been devised, so although the patterns being imaged varied, this procedure of setting up axes generally 
worked. We then proceeded to take a series of images, each 640 pixels by 480 pixels, with a 20 pixel overlap, of the detector. This resulted in roughly ten thousand images per detector.

Once the detector had been imaged, the images needed to be compiled into a larger image that one can browse through easily and detect flaws. During this process, for which we used the Java programming language, we could account for small corrections to the imaging process, such as offset, overlap, and rotation (the rotation of the camera was not necessarily aligned with the coordinate system of the detector). Compiling all the images into a single large image took significantly more memory than most computers have, so they were arranged together into larger tiles, each several thousand pixels wide. Any overlap between images was saved as an average of the overlapped areas. Instead of rotating the individual images and putting them together, we rotated the coordinate system and kept the images in their own coordinate system to prevent loss of quality (Figure 4). Blank tiles were not written to save space and processing time.

In the stitching together of the images, we also implemented a technique known as flat field averaging, used to remove systematic variation in light intensity from a non-uniform light source. This procedure involves averaging several blank images (containing no features), and dividing every pixel by the average pixel value of the averaged image. This matrix can then be divided into all other images to remove systematic variations. In color images like the ones worked with here, this needs to be done separately for each color channel.

After the large composite images were created, a navigator needed to be created to view them for the purpose of finding hair-line fractures and other surface flaws. For this we used Ben Legler's image viewer[5] and the Google maps API[6] as a foundation. The Google API takes in many small image tiles and provides an interface through which the user can zoom, move, and measure distances. To make these small tiles we wrote a program to take the composite images created in the previous step and translate and scale them into smaller output tiles labeled correctly (<zoom level $\left.\rangle_{-}\langle\text {column }\rangle_{-}\langle\text {row }\rangle_{\text {._filetype }}\right\rangle$ ) along with the 
.txt file needed for the Google API.

Processing these images took a long time, so we implemented multi-core threading to split the program between multiple computers or cores. This was done by splitting the rows being processed into different groups for the different processors. As input, we gave the program the number of processes desired for the split, $N$, as well as which process is to be run by each call (thread number), $n$. The program then ran on the rows defined in the range given by:

$$
\operatorname{ceil}\left(\frac{n}{N} \lambda\right)<\operatorname{row}<\operatorname{floor}\left(\frac{n+1}{N} \lambda\right)
$$

where $\lambda$ is the total number of rows of images produced. Empty tiles are deleted to save space and processing time.

\section{RESULTS}

In short, the programs we wrote worked well, and produced a useful navigation tool. Each set of $8,000-9,000$ images taken by the detector was stitched together with smooth looking interfaces that were barely noticeable. Everything was rotated and offset properly so lines and features going through the images matched to adjacent images with no breaks. The process took roughly an hour to complete on an average modern processor, resulting in almost 15 images per tile, of which there are about 650 . Figure 5 shows an example tile of stitched images.

The tiling program took these programs and cut them into approximately 85,000 tiles that worked well with the Google API. The web interface to navigate and view the detectors was easy to use and has proven an excellent tool for examining the detectors. The zoom and pan was smooth and loaded fast, and the additional tools such as the ruler worked perfectly. The tile creation process took around ten to thirteen total run hours, which was 
split between two cores. A sample interface can be seen at http://www.slac.stanford. edu/exp/cdms/CDMS_ImageNavigatorII/IZipG47_Side1_TES/.

The flat field averaging had a drastic effect on the smoothness seen across the images. Figure 5 shows the difference between tiles where flat field averaging was implemented and where it was not. Because the brightness varied across each image, the lines where the images were stitched together can be clearly seen. In the image where flat fielding is implemented, the systematic lighting differences were taken out and the resulting composite image is smooth and seamless.

\section{DISCUSSION AND CONCLUSIONS}

Although the programs worked well in their purpose, the image sets are large and it took many hours to process them. As the program has to be run after every imaging, improvements in speed would be exceptionally valuable. In the future the results from these programs can also be integrated with software for detecting flaws. For example, software could be written to compare the stitched images to the corresponding sections of the template using a threshold to match the colors. Any differences, where flaws are most likely to be, could then be highlighted and overlaid on the detector using the navigator so one can easily see where flaws and corrections are. This flaw detection is crucial to the overall workings of the detector. The detector needs to be as accurate and precise as possible to isolate dark matter detections from background. Determining flaws in the detectors and making any possible repairs to these areas is an important part of the experiment. Currently the detectors need to be completely examined by hand after each step in the photolithography process. Using automated detection of flaws would allow people to only examine the necessary small areas, resulting in an increase in production speed and scalability of detector fabrication. With 100 or more detectors to be fabricated, this will have a drastic effect on the timing of the 
experiment.

\section{ACKNOWLEDGMENTS}

I would like to thank Richard Partridge as my primary mentor throughout the process. Thanks is also due to Rudy Resch and the rest of the CDMS group for advice and suggestions, as well as SLAC National Accelerator Laboratory and the United States Department of Energy for making this experience possible.

\section{APPENDIX}

The input.txt file contains parameters needed to run the programs. Comments can be set using the pound symbol (\#). Below are listed the parameters used by the programs. Each parameter should be stated on a new line, with the name of the parameter separated from its value by a tab.

image_path The directory path where all the images taken with the OGP are stored.

save_path The path to the directory in which all the folders described below reside, including web files.

autorun_file The name of the autorun file (which should be in the image_path) which created the images.

image_name The name of the image files, with the number of the image replaced by a modulus (\%).

theta The angle between the coordinate systems of the camera and the detector, in radians. pixel_size The metric length in $\mathrm{mm}$ of each pixel. For a x75.3 magnification, this is $5.452 \mathrm{um}$, and for $\mathrm{x} 293$ magnification, it is $1.397 \mathrm{um}$.

blanks The numbers of the blank images used for the FF averaging, separated by commas. 
tile_width The width of each composite tile created.

tile_height The height of each composite tile created.

There is a precise file structure that should be followed for running Stitcher.java and Tiler.java. Although the image files and the autorun file from the OGP can be stored anywhere, as long as they are in the directory specified by image_path, the following files should be included in the save_path directory:

index.htm The html file that creates the online navigator.

input.txt The input file where the parameters above are defined by the user.

resources This folder contains the files needed for the navigator, and should not be edited unless to change the interface.

stitched_images Images created by Stitcher will be saved here.

tiles The tiles created by Tiler for the Google API will be saved to this folder.

\section{REFERENCES}

[1] F. Zwicky, "Spectral displacement of extra galactic nebulae," Helv. Phys. Acta, 6, pp. 110-127, 1933.

[2] D. Clowe et al., "A direct empirical proof of the existence of dark matter." Astrophysics Journal, 2006.

[3] J. P. Filippini, "A search for wimp dark matter using the first five-tower run of the cryogenic dark matter search," Ph.D. dissertation, (University of California, Berkeley), 2008. 
[4] S. W. Leman, "Developement of phonon-mediated transition-edge-sensor x-ray detectors for use in astronomy," Ph.D. dissertation, Stanford University, September 2006.

[5] B. Legler. Gmap image viewer. http://www.rmh.uwyo.edu/gmapviewer/about.php.

[6] Google maps javascript api. http://code.google.com/apis/maps/documentation/ javascript/.

[7] (2008, August) The matter of the bullet cluster. http://apod.nasa.gov/apod/ap080823. html.

[8] NASA/CXC/M.Weiss. 4-panel illustrations of cluster collisions. http://chandra.harvard. edu/photo/2006/1e0657/more.html. 


\section{FIGURES}

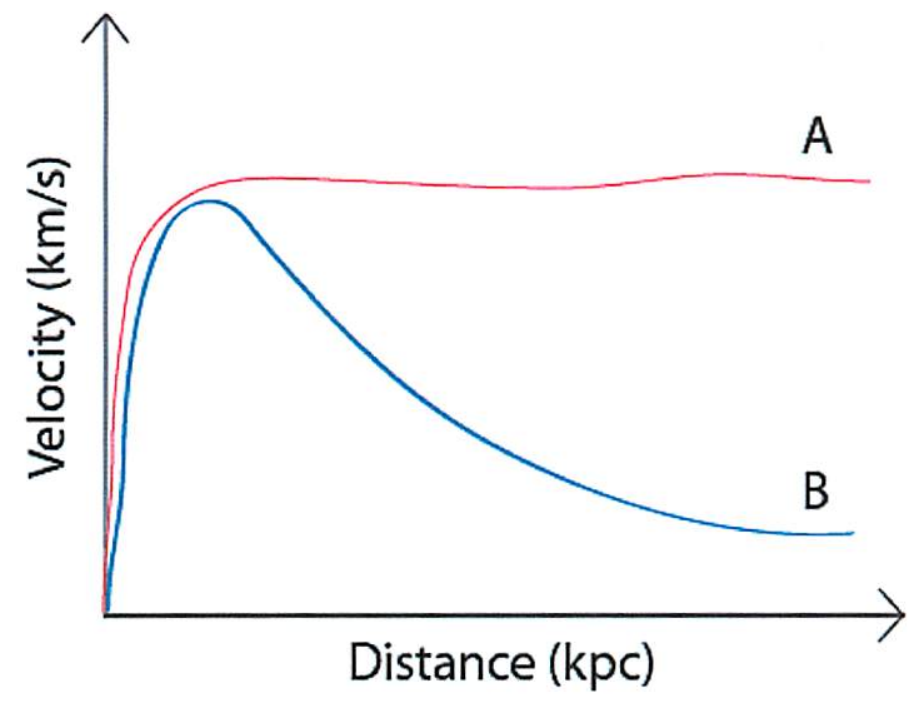

Figure 1: An illustration of the expected and measured rotation curves of the galaxy, showing the average velocity of matter as a function of distance from the center of the galaxy. Assuming Keplerian motion, and the fact that visible matter is concentrated at the center of the galaxy, we would expect rotational velocity to decrease with radius (curve B). The measured relationship (curve A) suggests that a large source of non-light-emitting matter exists in the galaxy or that Newtonian mechanics has flaws. 

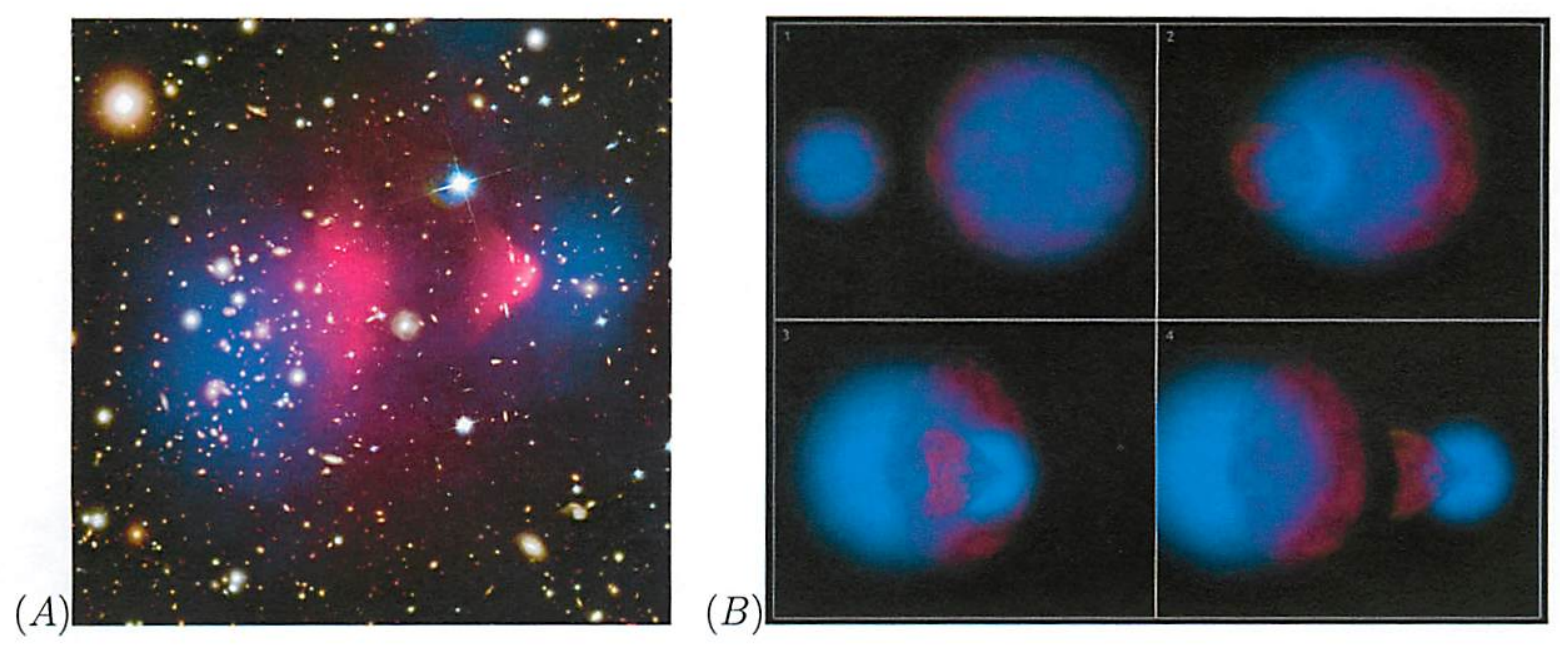

Figure 2: (A) An image of the bullet cluster taken with the Hubble telescope, with an overlay showing the calculated mass distribution in blue.[7] (B) Computer simulations show that a model with weakly interacting dark matter closely match what we see.[8]

$(A)$
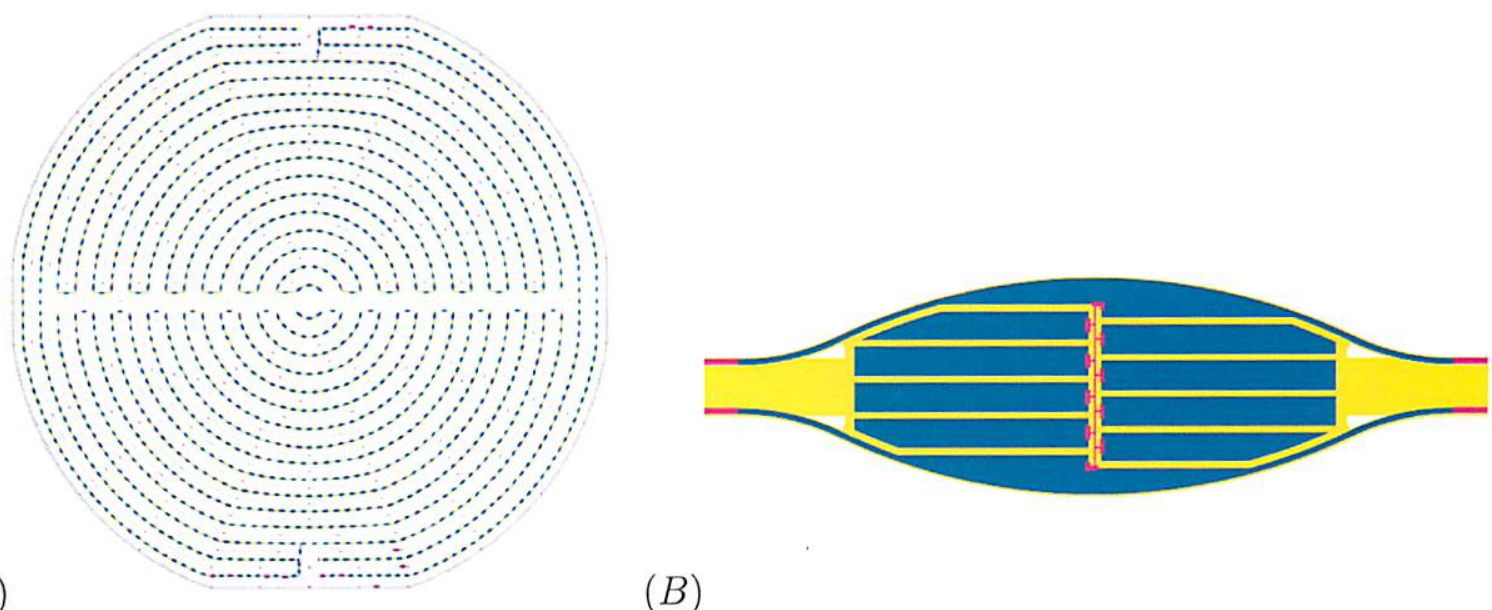

Figure 3: (A) The ends of the spherical germanium detector have nodes arranged in four areas (top, bottom, and two outer) to help with triangulation of the phonons they detect. (B) Phonons break the cooper pairs in the aluminum (blue), which cause a measurable change in current through the tungsten (pink). 


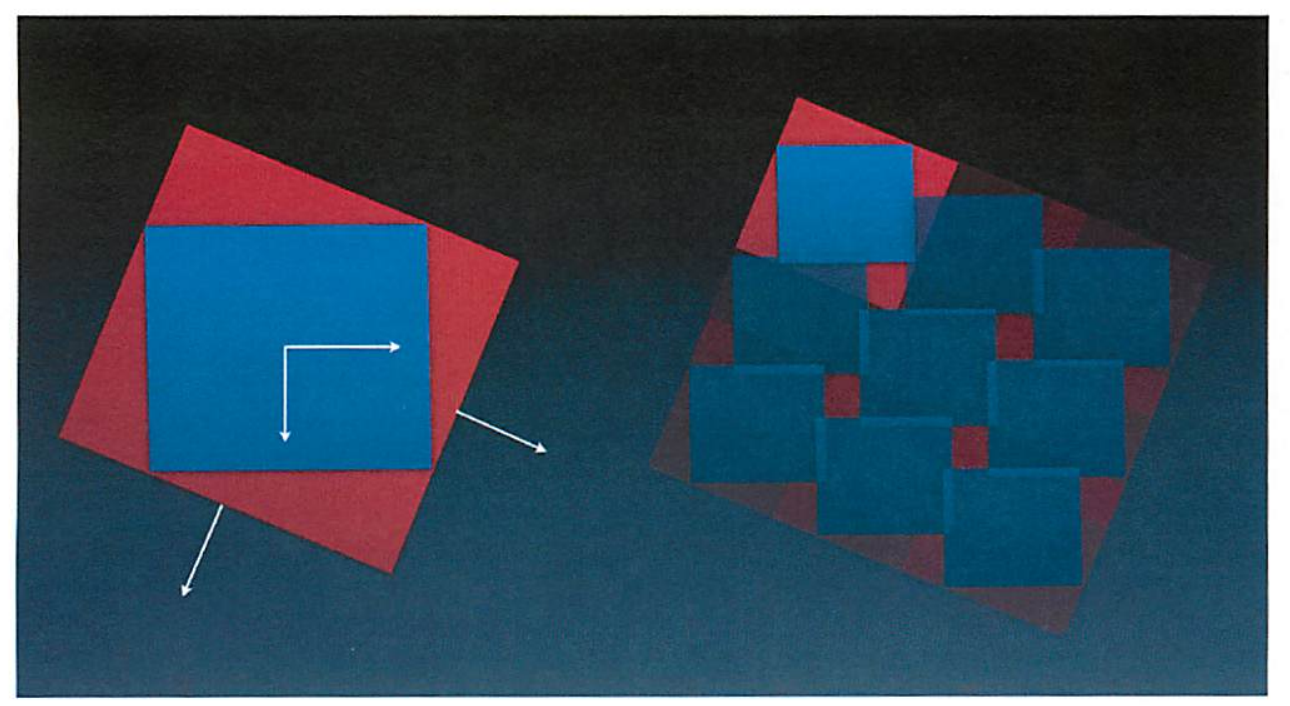

Figure 4: Instead of rotating each image (blue) within the reference frame of the detector (red), we rotated the reference frame of the detector to prevent loss of quality. 


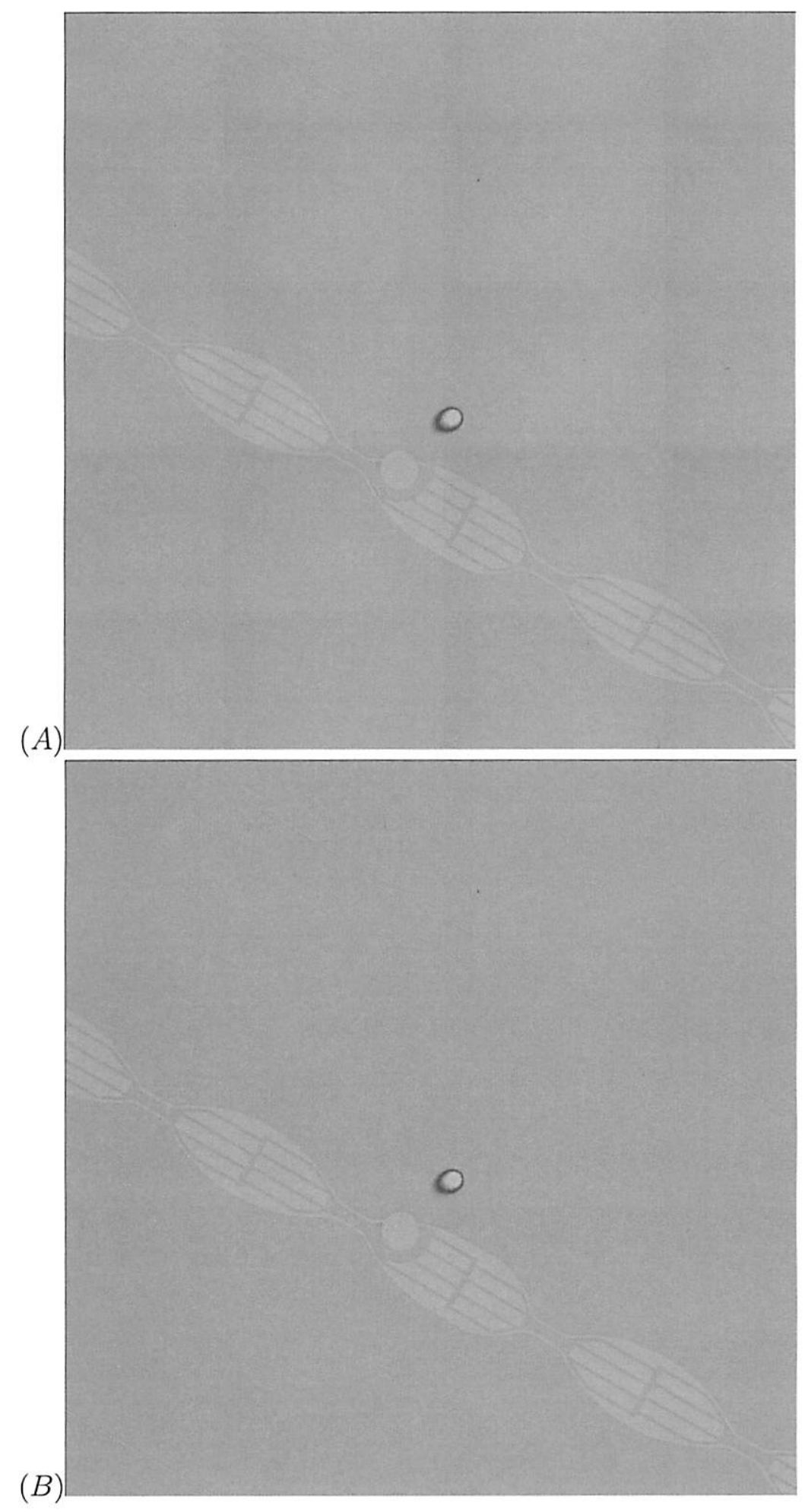

Figure 5: (A) These images of the detector were put together without flat field averaging. The seams where they were stitched together are visible because of the variation in lighting across the image. (B) Using flat fielding, thes@ systematic variations can be removed, creating a much smoother looking image. Note: the difference in contrast is not caused by the flat fielding. 